\title{
Generation and characterization of a monoclonal antibody against MERS-CoV targeting the spike protein using a synthetic peptide epitope-CpG-DNA-liposome complex
}

\author{
Byoung Kwon Park ${ }^{1}$, Sony Maharjan ${ }^{1}$, Su In Lee ${ }^{1}$, Jinsoo Kim ${ }^{2}$, Joon-Yong Bae ${ }^{3}$, Man-Seong Park ${ }^{3}$ E Hyung-Joo Kwon ${ }^{1,2, *}$ \\ ${ }^{1}$ Center for Medical Science Research, College of Medicine, Hallym University, Chuncheon 24252, ${ }^{2}$ Department of Microbiology, College of \\ Medicine, Hallym University, Chuncheon 24252, ${ }^{3}$ Department of Microbiology, College of Medicine, and the Institute for Viral Diseases, \\ Korea University, Seoul 02841, Korea
}

\begin{abstract}
Middle East respiratory syndrome coronavirus (MERS-CoV) uses the spike (S) glycoprotein to recognize and enter target cells. In this study, we selected two epitope peptide sequences within the receptor binding domain (RBD) of the MERS-CoV S protein. We used a complex consisting of the epitope peptide of the MERS-CoV $S$ protein and CpG-DNA encapsulated in liposome complex to immunize mice, and produced the monoclonal antibodies 506-2G10G5 and 492-1G10E4E2. The western blotting data showed that both monoclonal antibodies detected the $S$ protein and immunoprecipitated the native form of the $S$ protein. Indirect immunofluorescence and confocal analysis suggested strong reactivity of the antibodies towards the $S$ protein of MERS-CoV virus infected Vero cells. Furthermore, the 506-2G10G5 monoclonal antibody significantly reduced plaque formation in MERS-CoV infected Vero cells compared to normal mouse IgG and 492-1G10E4E2. Thus, we successfully produced a monoclonal antibody directed against the RBD domain of the $S$ protein which could be used in the development of diagnostics and therapeutic applications in the future. [BMB Reports 2019; 52(6): 397-402]
\end{abstract}

\section{INTRODUCTION}

Middle East respiratory syndrome coronavirus (MERS-CoV) is a fatal zoonotic pathogen that causes acute respiratory disease, MERS, in humans (1-3). MERS-CoV, a lineage C betacoronavirus, first appeared in Saudi Arabia in June 2012 (3-5). Since then, the cases of MERS-CoV human infection have been

*Corresponding author. Tel: +82-33-248-2635; Fax: +82-33-2413640; E-mail: hjookwon@hallym.ac.kr

https://doi.org/10.5483/BMBRep.2019.52.6.185

Received 8 August 2018, Revised 5 September 2018, Accepted 11 October 2018

Keywords: B cell epitope, Lipoplex (O), MERS-CoV, Monoclonal antibody, Spike protein reported to the World Health Organization (WHO) from 27 countries, mostly from Middle East countries (6). Globally, as of May 2018, MERS-CoV has caused a total of 2,220 cases of infections with at least 790 individual deaths (mortality rate: $35.6 \%$ ), with majority being reported from Saudi Arabia (1844 cases with 716 deaths) (6). Another major outbreak outside of the Middle East took place in South Korea in 2015 resulting in 186 cases with 36 fatalities $(7,8)$. An elevation in the pathogenicity of MERS-CoV and absence of effective vaccines or therapeutics against the virus could be a risky combination inviting a pandemic in the future. Therefore, ideal and effective therapeutic measures for the prevention and treatment of MERS are urgently required.

Previous reports have shown neutralizing monoclonal antibodies as potential candidates for the treatment of numerous diseases including virus infections, immune dysfunction and cancer (2, 9, 10). The MERS-CoV genome consists of structural proteins such as the spike (S), membrane $(\mathrm{M})$, envelope (E) and nucleocapsid $(\mathrm{N})(2,11)$. The $S$ glycoprotein of MERS-CoV portrays a crucial role in viral attachment and subsequent entrance into host cell $(12,13)$. The $\mathrm{S}$ glycoprotein is comprised of two subunits: receptor recognizing S1 and membrane fusion S2 $(12,13)$. The S1 subunit of MERS-CoV contains the receptor binding domain (RBD) which is responsible for interacting with the cellular receptor dipeptidyl peptidase 4 (DPP4; also called CD26) in the host cell membrane $(13,14)$. The heptad repeat (HR) 1 and 2 regions of the S2 subunit mediate fusion between the viral and host cell membranes (12). Due to the vital antigenic property, the S protein has been focus of the development of therapeutic strategies against MERS $(2,11)$. Importantly, antibody production against the RBD has been a functional determinant factor for promising diagnostic and therapeutic targeting (2).

We previously generated antibodies against hepatitis $C$ virus (15), influenza virus (16), respiratory syncytial virus (17) and MERS-CoV M protein (18) by immunization with a complex of a B cell epitope peptide coencapsulated with CpG-DNA in a dioleoyl phosphatidylethanolamine (DOPE): cholesterol hemi-

ISSN: 1976-670X (electronic edition)

Copyright (c) 2019 by the The Korean Society for Biochemistry and Molecular Biology

(c) This is an open-access article distributed under the terms of the Creative Commons Attribution Non-Commercial License (http://creativecommons.org/licenses/by-nc/4.0) which permits unrestricted non-commercial use, distribution, and reproduction in any medium, provided the original work is properly cited. 
ssuccinate (CHEMS) complex (named as Lipoplex (O)). In this study, we selected novel B cell epitope peptide sequences, Spike-492 and Spike-492 (L506F), from the MERS-CoV S protein-RBD of representative the South Korean and Saudi Arabian strains, respectively. The $B$ cell epitope peptide sequence, Spike-492 (L506F), has a single amino acid substitution of phenylalanine for leucine at residue 506 . Herein, we generated monoclonal antibodies, 506-2G10G5 and 492-1G10E4E2, specific against the $S$ protein of MERS-CoV by immunizing mice with a complex of the $B$ cell epitope peptide and Lipoplex (O). Our data show the potential of the 506-2G10G5 monoclonal antibody for diagnostic and therapeutic use against emerging MERS-CoV infection.

\section{RESULTS}

\section{Analysis of the B cell epitope and production of the antibody} targeting the $S$ protein epitope of MERS-CoV

$B$ cell epitope identification and selection are one of the most important factors in epitope-based antibody production. Hence, candidate B cell epitopes from the amino acid sequence of the MERS-CoV S protein were predicted using the Immune Epitope Database and Analysis Resources (IEDB) tool based on the epitope prediction, surface accessibility and antigenicity scale (http://tools.iedb.org/bcell). Because the RBD
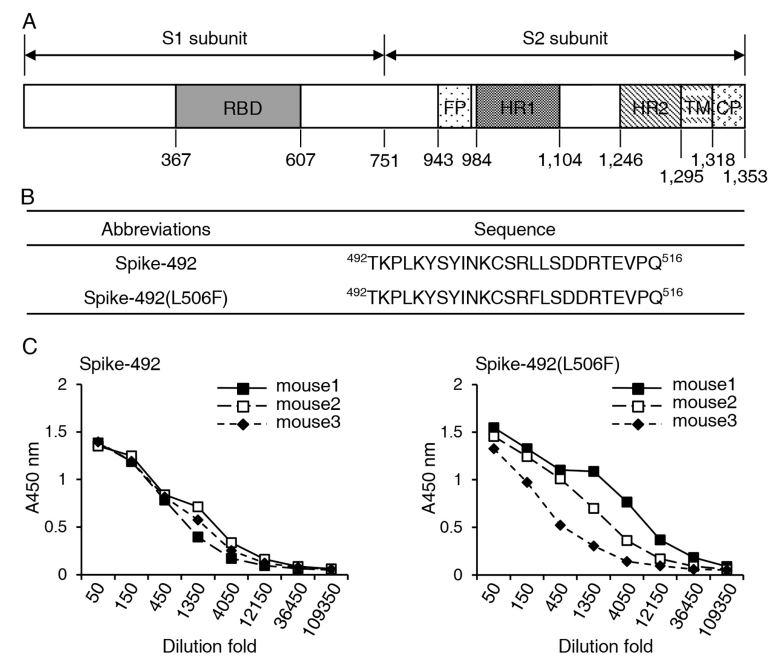

Fig. 1. Production of the B cell epitope-specific antibody. (A) Schematic representation of the MERS-CoV $\mathrm{S}$ protein. The $\mathrm{S}$ protein consists of S1 and S2 subunits. RBD, receptor binding domain; FP, fusion peptide; HR1 and HR2, heptad repeat region 1 and 2; TM, transmembrane; CP, cytoplasmic tail. (B) Selection of the B cell epitope peptide sequence using the IEDB based on the epitope prediction, surface accessibility and antigenicity index. (C) ELISA analysis showing the generation of MERS-CoV Spike-492- or Spike-492 (L506F)-specific antibodies using sera. Sera were isolated from BALB/C mice immunized with a complex of B cell epitope peptide (Spike-492 or Spike-492 (L506F)) and Lipoplex (O). domain within the $S$ protein is responsible for binding to the host cell $(2,18)$, the Spike-492 and Spike-492 (L506F) peptide sequences corresponding to the 492th-516th amino acid residues within the RBD domain of MERS-CoV S protein were selected and synthesized (Fig. 1A and B). To determine the efficiency of the peptides as a B cell epitope, each peptide and the Lipoplex (O) complex were formulated and then immunized into the BALB/C mice. To screen the antibody titers, ELISA was performed using the sera of the immunized mice. Both of the peptides induced a robust production of peptide-specific IgGs (Fig. 1C). Thus, the immunogenic epitope peptides were successfully designed and produced.

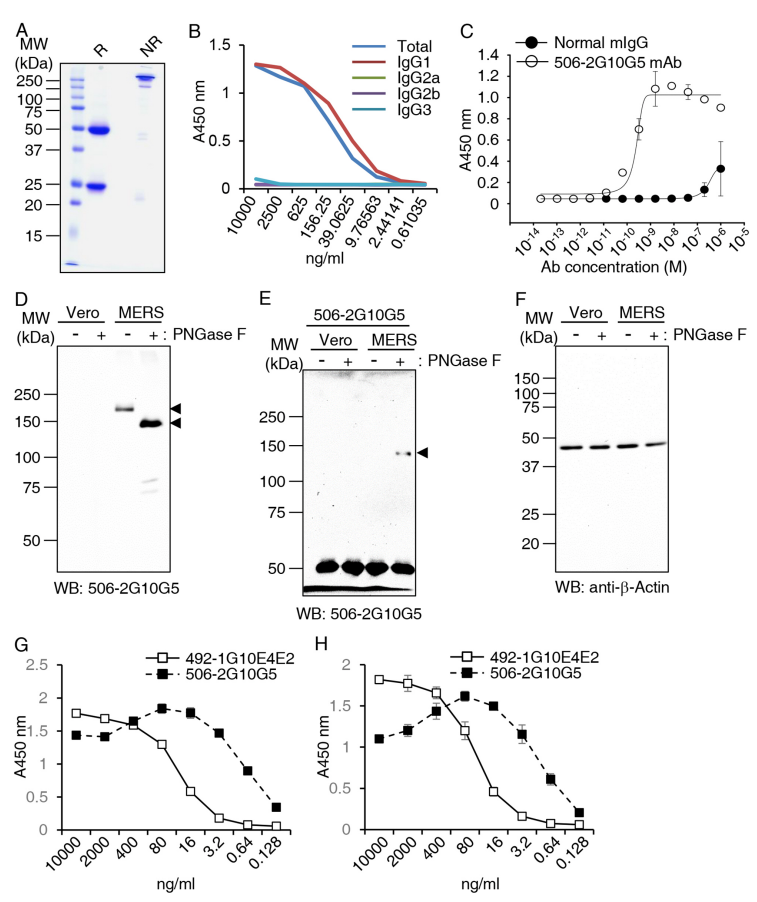

Fig. 2. Purification and characterization of anti-MERS-CoV Spike-492 (L506F) epitope-specific monoclonal antibody. (A) Purified 506-2G10G5 monoclonal antibody was analyzed by SDS-PAGE. $R$ reducing condition; NR, non-reducing conditions. (B) Isotype of the purified 506-2G10G5 monoclonal antibody was determined by ELISA. (C) The binding affinity of the 506-2G10G5 monoclonal antibody to the MERS-CoV Spike-492 (L506F) peptide was measured by ELISA, and the $\mathrm{EC}_{50}$ value was evaluated with the Sigma Plot program. (D-F) MERS-CoV-infected and non-infected Vero cells lysates were treated with PBS $(-)$ or PNGase F $(+)$. The lysates were immunoblotted with the 506-2G10G5 monoclonal antibody (D). Western blot analysis using the 506-2G10G5 monoclonal antibody was performed following the immunoprecipitation of lysates with the 506-2G10G5 monoclonal antibody (E). Lysates were subjected to western blotting with an anti- $\beta$ actin antibody $(\mathrm{F})$. $(\mathrm{G}, \mathrm{H})$ Cross-reactivity of the monoclonal antibodies. 96-well immunoplates coated with the MERS-CoV Spike-492 (G) or Spike-492 (L506F) (H) peptides and incubated with either the 492-1G10E4E2 or 506-2G10G5 monoclonal antibody. The reactivity of the antibodies to each peptide was determined by ELISA assay. 
Production and characterization of the monoclonal antibody specific to the Spike-492 (L506F) or Spike-492 epitope of MERS-CoV

A potential and positive monoclonal antibody producing 506-2G10G5 clone and 492-1G10E4E2 clone were generated from the fusion with SP2/0 myeloma cells and splenocytes from the mice immunized with the Spike-492 (L506F) or Spike-492 peptide and Lipoplex (O) complex. To obtain the monoclonal antibody on a large scale, ascitic fluid was collected from the mice injected with the 506-2G10G5 clone (Fig. 2A) or 492-1G10E4E2 clone (Fig. S1A), and the antibodies were purified. Next, the isotypes of the 506-2G10G5 and 492-1G10E4E2 monoclonal antibodies were determined by ELISA and found to be $\operatorname{lgG} 1$ and $\operatorname{IgG} 2 \mathrm{a}$, respectively (Fig. 2B and Fig. S1B). The binding affinities of the monoclonal antibodies targeting the Spike-492 (L506F) peptide and Spike-492 was assessed by ELISA, and the EC50 values of the monoclonal antibodies 506-2G10G5 and 492-1G10E4E2 were determined to be $178 \mathrm{pM}$ and $3.3 \mathrm{nM}$, respectively (Fig. 2C and Fig. S1C).

\section{Detection of the MERS-CoV S protein by the monoclonal antibody specific to the Spike-492 (L506F) or Spike-492 epitope of MERS-CoV}

To further characterize whether the monoclonal antibody 506-2G10G5 or 492-1G10E4E2 recognizes the S protein of MERS-CoV, western blotting and immunoprecipitation were performed with MERS-CoV-infected and non-infected Vero cells. The western blotting results (Fig. 2D, Fig. S1D and Fig. S2A) show that both monoclonal antibodies detected a protein band corresponding to the $\mathrm{S}$ protein in the molecular weight range in the MERS-CoV-infected Vero cells, however, no band was observed in the non-infected Vero cells. Treatment with peptide-N-glycosidase (PNGase $\mathrm{F}$ ) resulted in the reduction of the apparent molecular weight of the $S$ protein band compared with the untreated sample (Fig. 2D and Fig. S1D) suggesting that both monoclonal antibodies could recognize the $S$ protein in its glycosylated and de-glycosylated form. Furthermore, immunoprecipitation was done to evaluate if the monoclonal antibodies could recognize the $S$ protein in its native form. Both monoclonal antibodies immunoprecipitated the native form of the $S$ protein from MERS-CoV-infected Vero cells lysates (Fig. S1E and Fig. S2B). In addition, both monoclonal antibodies recognized the de-glycosylated protein band in the PNGase F treated immunoprecipitated lysate (Fig. 2E and Fig. S1E). To further determine the cross-reactivity of the 506-2G10G5 and 492-1G10E4E2 monoclonal antibodies with each corresponding epitope, ELISA was performed. The 506-2G10G5 monoclonal antibody showed remarkable cross-reactivity to Spike-492 peptides which was higher than the result shown by the 492-1G10E4E2 monoclonal antibody to Spike-492 (L506F) (Fig. 2G and H). Therefore, both of the antibodies exhibited specific binding to the MERS-CoV $\mathrm{S}$ protein.
Reactivity of the 506-2G10G5 or 492-1G10E4E2 monoclonal antibody to the $S$ protein in the MERS-CoV-infected cells To further confirm the reactivity of the 506-2G10G5 or 492-1G10E4E2 monoclonal antibody, we performed indirect immunofluorescence assay (IFA) in non-infected and MERS-CoV-infected Vero cells. Fluorescence microscopy showed the strong fluorescence signal in the virus infected cells incubated with either of the monoclonal antibodies, whereas no fluorescence was observed with the normal mouse IgG or secondary antibody control (Fig. 3A and Fig. S3A). To further substantiate the specificity of both monoclonal antibodies to the $\mathrm{S}$ protein of MERS-CoV, confocal microscopy was performed. MERS-CoV-infected or non-infected Vero cells were stained with the normal mouse IgG or the 506-2G10G5 or 492-1G10E4E2 monoclonal antibody. The confocal microscopy images of clearly show the fluorescence signal within the cytosolic region of the MERS-CoV infected cells stained with the 506-2G10G5 or 492-1G10E4E2 monoclonal antibody. No staining was observed in cells incubated with the

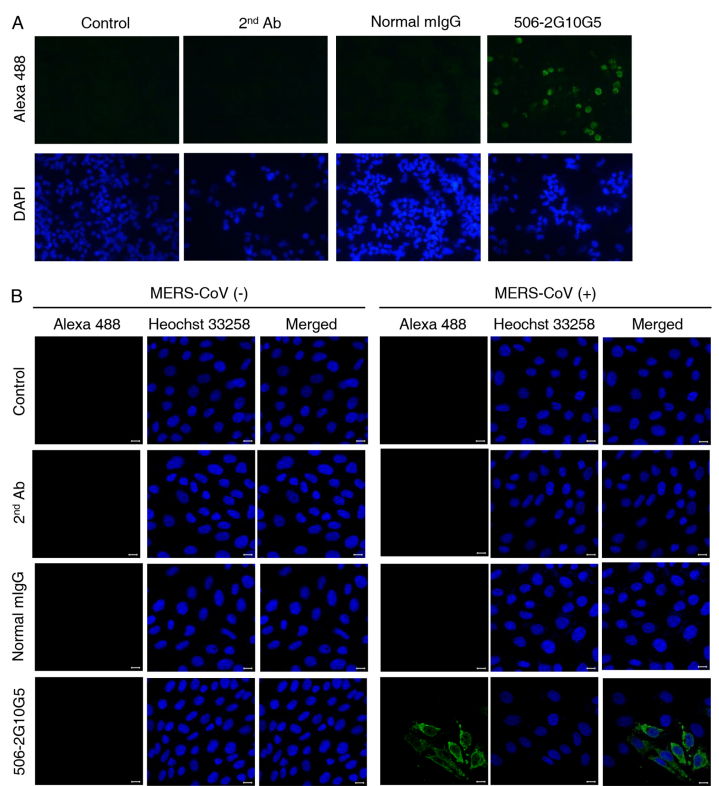

Fig. 3. Immunofluorescence assay and confocal microscopy for the detection of MERS-CoV-infected cells with the Spike-492 (L506F) monoclonal antibody. (A) Indirect immunofluorescence assay. A mixture of MERS-CoV-infected and non-infected Vero cells was stained with the 506-2G10G5 monoclonal antibody or normal mouse IgG, followed by incubation with the Alexa488-conjugated goat anti-mouse IgG antibody (green). Cells were counterstained with Hoechst 33258 for nuclear staining (blue). Images were taken using a fluorescence microscope. (B) Confocal microscopy. Vero cells were infected with MERS-CoV for 2 days. The cells were stained with the 506-2G10G5 monoclonal antibody or normal mouse IgG and then incubated with the Alexa488conjugated goat anti-mouse IgG antibody (green). Nuclei were stained using Hoechst 33258 (blue). Scale bar, $10 \mu \mathrm{m}$. 
normal mouse IgG or secondary antibody control (Fig. 3B and Fig. S3B). These results demonstrate that both monoclonal antibodies efficiently recognize the MERS-CoV $S$ protein in MERS-CoV-infected cells.

\section{6-2G10G5 monoclonal antibody inhibited MERS-CoV infection in Vero cells}

Given the specificity and binding affinity of the 506-2G10G5 or 492-1G10E4E2 monoclonal antibody towards the $S$ protein of MERS-CoV, we investigated the inhibitory activities of both monoclonal antibodies against MERS-CoV infecting using a plaque reduction assay. In this assay, both monoclonal antibodies inhibited plaque formation when compared to the nomal mouse IgG in a concentration-dependent manner. However, better inhibition of plaque formation was observed when treated with the 506-2G10G5 monoclonal antibody compared with the 492-1G10E4E2 monoclonal antibody (Fig. $4 \mathrm{~A}$ and $\mathrm{B})$. Thus, the results demonstrate the efficacy of the 506-2G10G5 monoclonal antibody, signifying its potential therapeutic applications against MERS-CoV infection.

\section{DISCUSSION}

It has been demonstrated that MERS-CoV infection can be severe health hazard to mankind worldwide $(1,3)$. As of today, effective therapeutics against MERS-CoV infection are unavailable which contributes to its high mortality rate (2). Clearly, an effective approach for neutralizing the viral infection is needed. In recent years, there has been an increase in the development of monoclonal antibodies for the effective treatment of various infectious viruses such as rabies, HIV, SARS-CoV, influenza, and Ebola $(9,19)$. Here, we produced and characterized the specificity and functionality of a

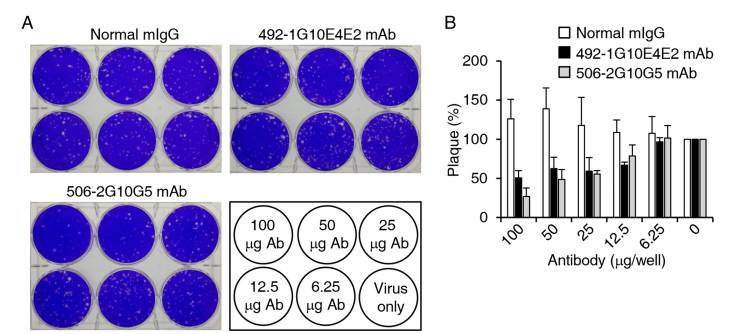

Fig. 4. Inhibition of MERS-CoV infection by the 492-1G10E4E2 and 506-2G10G5 monoclonal antibody. MERS-CoV was preincubated with two fold serially diluted normal mouse IgG, 492-1G10E4E2 or 506-2G10G5 monoclonal antibody for $30 \mathrm{~min}$ at $37^{\circ} \mathrm{C}$. The virus-antibody mixture was added to the Vero cells and incubated for $1 \mathrm{~h}$. After the incubation, the medium was replaced with DMEM/F12 containing $0.6 \%$ oxoid agar. The plaques were stained with crystal violet 4 days after infection. (A) A representative picture showing the plaque reduction assay. (B) Quatification of the plaque reduction assay against MERS-CoV after treatment with $100 \mu \mathrm{g} /$ well to $0 \mu \mathrm{g} /$ well of normal mouse IgG or 492-1G10E4E2 or 506-2G10G5 monoclonal antibody. monoclonal antibody against the $\mathrm{S}$ protein of MERS-CoV.

In previous studies, we successfully screened B cell epitope peptides and produced antibodies based on our own strategy using epitopes and CpG-DNA encapsulated with a DOPE: CHEMS complex as an adjuvant (20). In this study, we report generation and characterization of monoclonal antibodies against two selected B cell epitopes in the MERS-CoV S protein, Spike-492 and Spike-492 (L506F) peptides. Our data demonstrate that the hybridoma clones 492-1G10E4E2 and 506-2G10G5, obtained from mice immunized with the Spike-492 or Spike-492 (L506F) peptides and the Lipoplex (O) complex, produce antibodies specific to the MERS-CoV $\mathrm{S}$ protein and reciprocally cross-reactive to the epitopes representing the South Korean strains and the Saudi Arabian strains.

The efficacy of the antibodies were evaluated based on the binding affinity and neutralizing activity. Of the two monoclonal antibodies, 506-2G10G5 had a higher binding affinity to the Spike-492 and Spike-492 (L506F) peptides than that of the 492-1G10E4E2. In addition, 506-2G10G5 exhibited better neutralization activity than that of 492-1G10E4E2 demonstrated by the plaque reduction assay. Considering that the 506-2G10G5 monoclonal antibody is raised against the peptide sequence within the RBD domain, the antibody could have a potential therapeutic effect in addition to its diagnostic value. However, further experimental evidence is required to determine the therapeutic function of the antibody.

\section{MATERIALS AND METHODS}

\section{Cell line and virus}

Vero cells, African green monkey kidney cells, were obtained from the American Type Culture Collection (ATCC Inc.). Dulbecco's modified Eagle's medium (DMEM) purchased from Life Technologies (Thermo Fisher Scientific Co.) with supplementation of $10 \%$ fetal bovine serum (FBS, Thermo Fisher Scientific Co.), $25 \mathrm{mM}$ HEPES, $100 \mathrm{U} / \mathrm{ml}$ penicillin and $100 \mu \mathrm{g} / \mathrm{ml}$ streptomycin was used in the culture of Vero cells. The cells were incubated in an atmosphere of $5 \% \mathrm{CO}_{2}$ and $95 \%$ air at $37^{\circ} \mathrm{C}$. MERS-CoV/KOR/KNIH/002 052015 was obtained from the Korea Centers for Disease Control and Prevention (Permission No. 1-001-MER-IS-2015001).

\section{Preparation and synthesis of peptides}

The selection, analysis and synthesis of the B cell epitope peptides of the MERS-CoV $\mathrm{S}$ protein were performed as described previously (21). The B cell epitope peptide sequences for the MERS-CoV $\mathrm{S}$ protein were selected as Spike-492 $\left({ }^{492}\right.$ TKPLKYSYINKCSRLLSDDRTEVPQ $\left.{ }^{516}\right)$ from the MERS-CoV strain (MERS-CoV/KOR/KNIH/002 052015 (Gl: 829021049)) and Spike-492 (L506F) ( ${ }^{492}$ TKPLKYYSYINKCSRFL SDDRTEVPQ ${ }^{516}$ ) from the MERS-CoV strain (Spike glycoprotein universal sequence (Gl: 510785803)), and synthesized with an automated peptide synthesizer (Peptron III-R24, 
Peptron Inc.). A complex of the B cell epitope peptide and CpG-DNA (MB-ODN $4531(\mathrm{O})$ ) co-encapsulated in the DOPE: CHEMS (named as Lipoplex $(\mathrm{O})$ ) were prepared as previously described (17).

\section{Immunization of mice}

Four-week-old BALB/C $\left(\mathrm{H}-2^{\mathrm{b}}\right)$ female mice were purchased from Nara-Biotec Co. Ltd. The mice were maintained at an animal facility under specific pathogen-free conditions. All the experiments involving the animals were carried out with the approval from the Institutional Animal Care and Use Committee of Hallym University (Hallym2016-51). The mice were intraperitoneally immunized three times at 10-day intervals with $200 \mu \mathrm{l}$ of the Spike-492 peptide $(50 \mu \mathrm{g})$ or Spike-492 (L506F) peptide $(50 \mu \mathrm{g})$ and the Lipoplex (O) complex $(17,22,23)$.

\section{Monoclonal antibody production against the MERS-CoV S protein}

The monoclonal antibodies were produced in accordance with standard method as previously described $(24,25)$. Splenocytes isolated from the mice were fused with mouse SP2/0 cells (ATCC Inc.) in presence of polyethylene glycol solution (PEG, Sigma Chemical Co.). To acquire positive clones producing the MERS-CoV Spike-492- or Spike-492 (L506F)-specific monoclonal antibody, HAT medium (Sigma Chemical Co.) and HT medium (Sigma Chemical Co.) were used for the cultures and for the selection of hybridoma cells. For a large-scale production of the monoclonal antibodies, ascites fluid was collected from mice injected intraperitoneally with the selected hybridomas and purified by protein A column chromatography as previously described (17).

\section{ELISA assays}

To measure the epitope peptide-specific antibody titer, 96-well immunoplates (Thermo Fisher Scientific Co.) were coated with $5 \mu \mathrm{g} /$ well of the MERS-CoV Spike-492 or Spike-492 (L506F) peptides. The mice sera were obtained by retro orbital bleeding and analyzed as previously described (22). To identify the isotype of the monoclonal antibody, the HRPconjugated anti-mouse IgG (each isotype) antibody (Southern Biotechnology Associates Inc.) was used. For detection of cross-reactivity, 96-well immunoplates were coated with the MERS-CoV Spike-492 or Spike-492 (L506F) peptides and incubated with either 492-1G10E4E2 or 506-2G10G5 monoclonal antibody for $2 \mathrm{~h}$ before incubation with the secondary antibody. The absorbance was evaluated with the Spectra Max 250 microplate reader (Molecular Devices Co.) at $450 \mathrm{~nm}$ and then calculated with the SigmaPlot program to determine the affinity constant (EC50 value) as described previously (17).

\section{Western blotting and immunoprecipitation}

MERS-CoV-infected Vero cell lysates were run on SDS-PAGE and subsequently transferred onto a nitrocellulose membrane as described previously $(26,27)$, and detailed information is provided in the Supplementary Material.

\section{Peptide-N-glycosidase $\mathrm{F}$ digestion}

MERS-CoV-infected and non-infected Vero cells were lysed with lysis buffer $(0.5 \%$ SDS and $1 \% \beta$-mercaptoethanol) and boiled at $100^{\circ} \mathrm{C}$ for $10 \mathrm{~min}$. The lysates were then subjected to peptide-N-glycosidase $\mathrm{F}$ (PNGase $\mathrm{F}$ ) (Elpis Biotech Inc.) treatment at $37^{\circ} \mathrm{C}$ for $2 \mathrm{~h}$ and boiled at $100^{\circ} \mathrm{C}$ for $10 \mathrm{~min}$. After the digestion, the samples were detected with the 4921G10E4E2 or 506-2G10G5 monoclonal antibody by western blotting. For immunoprecipitation, the PNGase F-digested samples were incubated with the indicated antibodies overnight at $4^{\circ} \mathrm{C}$ followed by western blot analysis with the 492-1G10E4E2 or 506-2G10G5 monoclonal antibody.

\section{Indirect immunofluorescence and confocal microscopy}

For analysis of the indirect immunofluorescence assay, a mixture of MERS-CoV-infected and non-infected Vero cells at a ratio of 3:1 was seeded onto slide glasses. The cells were then fixed with acetone and incubated with the normal mouse lgG, 492-1G10E4E2 or 506-2G10G5 monoclonal antibody at $37^{\circ} \mathrm{C}$ for $2 \mathrm{~h}$. The samples were further incubated with Alexa Flour 488-conjugated goat anti-mouse IgG antibody (Thermo Fisher Scientific Co.). Finally, the samples were mounted and analyzed using a fluorescence microscope (1X70, Olympus Co.) $(28,29)$. To visualize the confocal microcopy, Vero cells $\left(5 \times 10^{4}\right)$ were seeded onto cover glasses in 12 well plates and infected with MERS-CoV $(0.1 \mathrm{MOI})$. After two days, the infected cells were fixed with $4 \%$ paraformaldehyde and subsequently blocked with $1 \%$ BSA and $0.1 \%$ triton X-100 in PBS. The slides were incubated in the presence of the 492-1G10E4E2 or 506-2G10G5 monoclonal antibody for $2 \mathrm{~h}$, washed and then incubated with the Alexa Flour 488conjugated goat anti-mouse $\operatorname{lgG}$ antibody for $1 \mathrm{~h}$. Hoechst 33258 (Thermo Fisher Scientific) was used to stain the nuclei. The slides were examined by Carl Zeiss LSM710 (Carl Zeiss Co. Ltd.).

\section{Plaque reduction assay}

$6 \times 10^{5}$ Vero cells/ well were plated on six-well plates (Thermo Fisher Scientific Co.) and cultured for $12 \mathrm{~h}$. Prior to infection, the MERS-CoVirus was pre-incubated with two fold serial diluted normal mouse IgG, 492-1G10E4E2 or 506-2G10G5 monoclonal antibodies for $30 \mathrm{~min}$ at $37^{\circ} \mathrm{C}$. The virus-antibody mixture was added to the Vero cells with $500 \mu$ l of PBS. After 1 $\mathrm{h}$ incubation, the supernatant was removed and $3 \mathrm{ml}$ of DMEMF12 medium (Thermo Fisher Scientific Co.) containing $0.6 \%$ oxoid agar were added. The plaques formed in each wells were stained with crystal violet (20) 4 days after infection. The plaques were counted and the percentage was calculated.

\section{ACKNOWLEDGEMENTS}

This work was supported by grants from the National Research 
Foundation (2016M3A9B6916708, 2009-0093812) funded by the Ministry of Science and ICT in the Republic of Korea. Byoung Kwon Park was supported by the Hallym University Postdoctoral Fellowship Program of 2017 (HLM-PF-2017-0001).

\section{CONFLICTS OF INTEREST}

The authors have no conflicting interests.

\section{REFERENCES}

1. Zumla A, Chan JF, Azhar El et al (2016) Coronaviruses drug discovery and therapeutic options. Nat Rev Drug Discov 15, 327-347

2. Du L, Yang Y, Zhou $Y$ et al (2017) MERS-CoV spike protein: a key target for antivirals. Expert Opin Ther Targets 21, 131-143

3. Mackay IM and Arden KE (2015) MERS coronavirus: diagnostics, epidemiology and transmission. Virol J 12, 222

4. Zaki AM, van Boheemen S, Bestebroer TM et al (2012) Isolation of a novel coronavirus from a man with pneumonia in Saudi Arabia. N Engl J Med 367, 1814-1820

5. Omrani AS, Al-Tawfiq JA and Memish ZA (2015) Middle East respiratory syndrome coronavirus (MERS-CoV): animal to human interaction. Pathog Glob Health 109, 354-362

6. World Health Organization, Middle East respiratory syndrome coronavirus (MERS-CoV), 2018. (http://www. who.int/emergencies/mers-cov/en/)

7. Ki M (2015) 2015 MERS outbreak in Korea: hospital-tohospital transmission. Epidemiol Health 37, e2015033

8. Durai P, Batool M, Shah M and Choi S (2015) Middle East respiratory syndrome coronavirus: transmission, virology and therapeutic targeting to aid in outbreak control. Exp Mol Med 47, e181

9. Lambour J, Naranjo-Gomez $M$, Piechaczyk $M$ and Pelegrin M' (2016) Converting monoclonal antibody-based immunotherapies from passive to active: bringing immune complexes into play. Emerg Microbes Infect 5, e92

10. Scott AM, Allison JP and Wolchok JD (2012) Monoclonal antibodies in cancer therapy. Cancer Immun 12, 14

11. Wang Q, Wong G, Lu G et al (2016) MERS-CoV spike protein: Targets for vaccines and therapeutics. Antiviral Res 133, 165-177

12. Gao J, Lu G, Qi J et al (2013) Structure of the fusion core and inhibition of fusion by a heptad repeat peptide derived from the $\mathrm{S}$ protein of Middle East respiratory syndrome coronavirus. J Virol 87, 13134-13140

13. Wang N, Shi X, Jiang L et al (2013) Structure of MERS-CoV spike receptor-binding domain complexed with human receptor DPP4. Cell Res 23, 986-993

14. Raj VS, Mou H, Smits SL et al (2013) Dipeptidyl peptidase 4 is a functional receptor for the emerging human coronavirus-EMC. Nature 495, 251-254

15. Kim D, Kwon HJ and Lee Y (2011) Activation of Toll-like receptor 9 and production of epitope specific antibody by liposome-encapsulated CpG-DNA. BMB Rep 44, 607-612

16. Rhee JW, Kim D, Park et al (2012) Immunization with a hemagglutinin-derived synthetic peptide formulated with a CpG-DNA-liposome complex induced protection against lethal influenza virus infection in mice. PLoS One 7, e48750

17. Park BK, Choi SH, Kim YE et al (2015) Monoclonal antibodies against the human respiratory syncytial virus obtained by immunization with epitope peptides and CpG-DNA-liposome complex. Monoclon Antib Immunodiagn Immunother 34, 101-109

18. Park BK, Lee SI, Bae JY et al (2018) Production of a Monoclonal Antibody targeting the $M$ protein of MERSCoV for detection of MERS-CoV using a synthetic peptide epitope formulated with a CpG-DNA-liposome complex. Int J Pept Res Ther https://doi.org/10.1007/s10989-0189731-8

19. Salazar G, Zhang N, Fu TM and An Z (2017) Antibody therapies for the prevention and treatment of viral infections. NPJ Vaccines 2, 19

20. Park BK, Park JY, Kim TH et al (2018) Production of an anti-TM4SF5 monoclonal antibody and its application in the detection of TM4SF5 as a possible marker of a poor prognosis in colorectal cancer. Int J Oncol 53, 275-285

21. Wu G, Maharjan S, Kim D et al (2018) A Novel monoclonal antibody targets mucin1 and attenuates growth in pancreatic cancer model. Int J Mol Sci 19, 2004

22. Park BK, Gautam A, Maharjan S et al (2017) Production of anti-c-Myc monoclonal antibody inhibiting DNA binding of c-Myc and max dimer by epitope peptide-CpGDNA-liposome complex without carriers. Int J Pept Res Ther https://doi.org/10.1007/s10989-017-9649-6

23. Wu G, Kim D, Kim JN et al (2018) A Mucin1 C-terminal subunit-directed monoclonal antibody targets overexpressed Mucin1 in breast cancer. Theranostics 8, 78-91

24. Kim D, Kwon S, Rhee JW et al (2011) Production of antibodies with peptide-CpG-DNA-liposome complex without carriers. BMC Immunol 12, 29

25. Yokoyama WM, Christensen M, Santos GD and Miller D (2006) Production of monoclonal antibodies. Curr Protoc Immunol 102, Unit 2.5

26. Maharjan S, Park BK, Lee SI et al (2018) Gomisin G inhibits the growth of triple-negative breast cancer cells by suppressing AKT phosphorylation and decreasing cyclin D1. Biomol Ther (Seoul) 26, 322-327

27. Maharjan S, Park BK, Lee SI et al (2018) Gomisin G suppresses the growth of colon cancer cells by attenuation of AKT phosphorylation and arrest of cell cycle progression. Biomol Ther (Seoul) https://doi.org/ 10.4062/biomolther.2018.054

28. Jo HS, Eum WS, Park EY et al (2017) Effects of PEP-1-FK506BP on cyst formation in polycystic kidney disease. BMB Rep 50, 460-465

29. Lee EW, Oh W, Song HP and Kim WK (2017) Phosphorylation of p53 at threonine 155 is required for Jab1-mediated nuclear export of p53. BMB Rep 50, 373-378 